\title{
Autoantibody profiles in systemic sclerosis; a comparison of diagnostic tests
}

Citation for published version (APA):

Alkema, W., Koenen, H., Kersten, B. E., Kaffa, C., Dinnissen, J. W. B., Damoiseaux, J. G. M. C., Joosten, I., Driessen-Diks, S., van der Molen, R. G., Vonk, M. C., \& Smeets, R. L. (2021). Autoantibody profiles in systemic sclerosis; a comparison of diagnostic tests. Autoimmunity, 54(3), 148-155.

https://doi.org/10.1080/08916934.2021.1907842

Document status and date:

Published: 03/04/2021

DOI:

10.1080/08916934.2021.1907842

Document Version:

Publisher's PDF, also known as Version of record

Document license:

Taverne

Please check the document version of this publication:

- A submitted manuscript is the version of the article upon submission and before peer-review. There can be important differences between the submitted version and the official published version of record.

People interested in the research are advised to contact the author for the final version of the publication, or visit the DOI to the publisher's website.

- The final author version and the galley proof are versions of the publication after peer review.

- The final published version features the final layout of the paper including the volume, issue and page numbers.

Link to publication

\footnotetext{
General rights rights.

- You may freely distribute the URL identifying the publication in the public portal. please follow below link for the End User Agreement:

www.umlib.nl/taverne-license

Take down policy

If you believe that this document breaches copyright please contact us at:

repository@maastrichtuniversity.nl

providing details and we will investigate your claim.
}

Copyright and moral rights for the publications made accessible in the public portal are retained by the authors and/or other copyright owners and it is a condition of accessing publications that users recognise and abide by the legal requirements associated with these

- Users may download and print one copy of any publication from the public portal for the purpose of private study or research.

- You may not further distribute the material or use it for any profit-making activity or commercial gain

If the publication is distributed under the terms of Article $25 \mathrm{fa}$ of the Dutch Copyright Act, indicated by the "Taverne" license above, 


\section{Autoimmunity \\ Autoimmunity}

\section{Autoantibody profiles in systemic sclerosis; a comparison of diagnostic tests}

Wynand Alkema, Hans Koenen, Brigit E. Kersten, Charlotte Kaffa, Jacqueline W. B. Dinnissen, Jan G. M. C. Damoiseaux, Irma Joosten, Sophie DriessenDiks, Renate G. van der Molen, Madelon C. Vonk \& Ruben L. Smeets

To cite this article: Wynand Alkema, Hans Koenen, Brigit E. Kersten, Charlotte Kaffa, Jacqueline W. B. Dinnissen, Jan G. M. C. Damoiseaux, Irma Joosten, Sophie Driessen-Diks, Renate G. van der Molen, Madelon C. Vonk \& Ruben L. Smeets (2021) Autoantibody profiles in systemic sclerosis; a comparison of diagnostic tests, Autoimmunity, 54:3, 148-155, DOI: 10.1080/08916934.2021.1907842

To link to this article: https://doi.org/10.1080/08916934.2021.1907842

曲 Published online: 05 Apr 2021.

Submit your article to this journal $\sqsubset$

Џll Article views: 215

Q View related articles $\widetilde{ }$

View Crossmark data $₫$ 


\title{
Autoantibody profiles in systemic sclerosis; a comparison of diagnostic tests
}

\author{
Wynand Alkema $^{\mathrm{a}, \mathrm{b}} \mathbb{D}$, Hans Koenen ${ }^{c *}$, Brigit E. Kersten ${ }^{\mathrm{d} *}$, Charlotte Kaffa ${ }^{\mathrm{e}}$, Jacqueline W. B. Dinnissen ${ }^{\mathrm{f}}$, \\ Jan G. M. C. Damoiseaux ${ }^{\mathrm{g}}$, Irma Joostenc, Sophie Driessen-Diks ${ }^{c}$, Renate G. van der Molen ${ }^{c *}$, \\ Madelon C. Vonk ${ }^{\mathrm{d} *}$ and Ruben L. Smeets ${ }^{\mathrm{c}, \mathrm{f}}$ \\ ${ }^{\mathrm{a}}$ Institute of Life Science and Technology, Hanze University of Applied Sciences, Groningen, The Netherlands; ${ }^{\mathrm{b}}$ TenWise B.V., Oss, The \\ Netherlands; 'Laboratory for Medical Immunology, Radboud University Medical Center, Nijmegen, The Netherlands; ${ }^{d}$ Department of

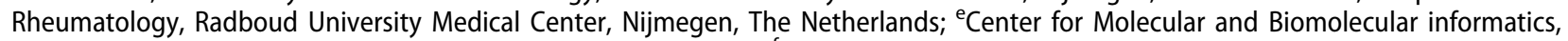 \\ Radboud University Medical Center, Nijmegen, The Netherlands; ${ }^{f}$ Radboudumc Laboratory for Diagnostics, Radboud University Medical \\ Center, Nijmegen, The Netherlands; ${ }^{9}$ Central Diagnostic Laboratory, Maastricht University Medical Center, Maastricht, The Netherlands
}

\begin{abstract}
Objectives: Autoimmune antibody profiling plays a prominent role in both classification and prognosis of systemic sclerosis (SSc). In the last years novel autoantibodies have been discovered and have become available in diagnostic assays. However, standardization in autoimmune serology is lacking, which may have a negative impact on the added value of autoantibodies in diagnosis and prognosis of SSc. In this paper we describe the comparison of commercially available diagnostic assays for the detection of SSc-associated autoantibodies and explored the coexistence of multiple SSc-associated autoantibodies within patients.

Methods: Serum samples of 347 patients from the Nijmegen Systemic Sclerosis Cohort were included in this study. All patients fulfilled the ACR/EULAR 2013 classification criteria for SSc and were classified as DCSSC or LCSSC according to the Leroy and Medsger criteria. All samples were evaluated on standard laboratory diagnostic tests for detection of SSc-specific autoantibodies CENPA and CENPB (ACA), Scl-70 (ATA), RNA Polymerase III (rp11/155) (ARA), and SSc-associated autoantibodies Fibrillarin, Th-To, PM-scl75, PM-Scl100, RNP68/A/C, Ku, NOR90, and PDGFR from suppliers EUROIMMUN, D-tek and Thermo Fisher Scientific.

Results: We found that $79 \%$ of the patients was positive for one or more of the SSc autoantibodies. Overall, a high agreement was observed between the diagnostic methods for the SSC-specific autoantibodies listed in the ACR/EULAR criteria (ATA, ACA, and ARA) (Cohen's kappa 0.53-0.97). However, a lower agreement was found for SSc-associated autoantibodies PM-Scl, and Ku, as well as for the SScspecific autoantibodies fibrillarin and Th-To. Furthermore, the data revealed that the presence of ATA, ARA and ACA is predominantly mutually exclusive, with only a fraction of the patients testing positive for both ATA and ARA.

Conclusion: Our data showed high concordance of prevalent SSc-specific autoantibodies between different diagnostic assays. Further standardisation for low prevalent SSc-specific and SSc-associated autoantibodies is needed.
\end{abstract}

\section{ARTICLE HISTORY}

Received 8 December 2020

Revised 12 February 2021

Accepted 21 March 2021

\section{KEYWORDS}

Autoantibody; diagnostic test; systemic sclerosis; clinical study; standardization

\section{Introduction}

Systemic sclerosis (SSc) is a chronic autoimmune disease characterized by inflammation, vasculopathy and fibrosis of the skin and internal organs. SSc can be subclassified in two major subtypes: limited cutaneous SSc (LcSSc) and diffuse cutaneous SSc (DcSSc), based on the extent of skin thickening [1]. DcSSc is associated with more severe organ involvement of the lungs and heart although in LcSSc this is not uncommon. Internal organ involvement is associated with a high morbidity and premature death [2]. To date, no cure is available for SSc but treatment with immunosuppressive drugs can increase the quality of life, decrease progression of organ involvement and increase survival [3]. SSc is a heterogeneous disease and therefore prediction of disease progression and complications is challenging. For both the patient and the clinician the uncertainty of the disease course and possible development of severe complications is a major concern. Although strong prognostic factors are lacking, autoantibodies have shown to be associated with disease course and occurrence of complications. Anticentromere (ACA), anti-topoisomerase, also called anti-Scl70 (ATA) and anti-RNA polymerase III (ARA) are the most prevalent autoantibodies and associated with a certain disease course and/or complication [4-6]. ACA is associated with LCSSc and pulmonary arterial hypertension (PAH) late in the disease course, whereas ATA is associated with

CONTACT Ruben L. Smeets ruben.smeets@radboudumc.nl Department for laboratory Medicine, Laboratory for Medical Immunology, Radboudumc, Geert Grooteplein noord 10, P.O. Box 9100, Nijmegen, 6500HB, The Netherlands

$*$ These authors have contributed equally to this work.

2021 Informa UK Limited, trading as Taylor \& Francis Group 
DcSSc, interstitial lung disease (ILD) and digital ulcera (DU) [7]. Patients with ARA antibodies more often have renal crisis, diffuse disease and gastric antral vascular ectasia (GAVE). For the autoantibodies with lower prevalence, the associations with disease phenotype are more difficult to demonstrate. However, some associations, were demonstrated in different studies. Anti-PM-Scl, anti-RNP68/A/C and anti-Ku are associated with overlap SSc and myositis [8]. Anti-fibrillarin autoantibodies are clinically associated with overlap SSc, myositis, joint involvement and $\mathrm{PAH}$ $[9,10]$. In the he ACR/EULAR classification criteria for SSc, the presence of ACA, ATA, and ARA contribute to the classification [11]. Since these autoantibodies are used for classification and are associated to prognosis and the occurrence of complications, it is of the utmost importance that available diagnostic assays have comparable results. Though immunoprecipitation is still perceived as the gold standard, this method is not standard of practice in most diagnostic laboratories. Line blot and enzyme linked immunoassays have shown to serve as a reliable alternative for the detection of autoantibodies. These more robust and less laborious diagnostic tests offer fast parallel (semi)quantitative detection of autoantibodies [12] Therefore, it is important to investigate and compare the performance of different commercially available test kits. Furthermore, knowledge about the test characteristics of the other SSc-specific and-associated antibodies is essential for appropriate interpretation of these test results.

\section{Materials and methods}

\section{Cohort enrolment}

This study was performed at the Radboud University Medical Centre, Nijmegen, the Netherlands, which is a tertiary referral centre for SSc in the Netherlands. Data of 347 consecutive patients from the Nijmegen Systemic Sclerosis Cohort were included in this study [13]. All patients fulfilled the ACR/EULAR 2013 classification criteria for SSc [11].

Patients were subclassified as having DcSSc or LcSSc according to the Leroy and Medsger criteria [1]. Informed consent was obtained from all patients and study protocol was reviewed and approved by the local ethical committee. Blood samples of patients were collected at scheduled outpatient clinic visits. Serum aliquots were stored at $-80^{\circ} \mathrm{C}$. Disease duration was defined as time from the first nonRaynaud phenomenon. We collected the main disease complications in our cohort namely: ILD, PAH, and DU. ILD was defined as more than $20 \%$ lung involvement (fibrosis/ ground glass) on High Resolution CT scan according to the Goh et al. algorithm [14]. PAH was diagnosed by right heart catheterization. DU is ulcers present distal from the PIP joint due to vasculopathy. Data from patients with current or past DU were collected. Because of the low prevalence of renal crisis, this data is not shown. Cardiac involvement is not displayed, as the diagnostics for this are currently under debate. Table 1 displays the baseline characteristics of the cohort.
Table 1. Baseline characteristics of the cohort.

\begin{tabular}{lcc}
\hline & DcSSc $(n=111)$ & LcSSc $(n=236)$ \\
\hline Age, mean (S.D.) & $57(11.6)$ & $60(12.3)$ \\
Female, $n$ (\%) & $65(59 \%)$ & $161(69 \%)$ \\
Disease duration in years (mean (SD) & $8.17(6.1)$ & $8.35(6.8)$ \\
Maximal mRSS, mean (S.D.) & $18.16(8.8)$ & $5.27(4.4)$ \\
ILD, $n(\%)$ & $60(54 \%)$ & $85(36 \%)$ \\
PAH, $n(\%)$ & $8(7 \%)$ & $35(15 \%)$ \\
DU, $n(\%)$ & $36(32 \%)$ & $82(35 \%)$ \\
\hline
\end{tabular}

DcSSc: diffuse cutaneous systemic sclerosis; LcSSc: limited cutaneous systemic sclerosis; mRSS: modified Rodnan Skin Score; PAH: pulmonary arterial hypertension; DU: digital ulcera; S.D.: standard deviation; IQR: interquartile range.

\section{Analysis for prevalent autoantibodies}

Sera were analyzed for prevalence of circulating autoantibodies using tests from three commercial suppliers, EUROIMMUN (Lübeck, Germany), D-tek (Mons, Belgium) and Thermo Fisher Scientific (Uppsala, Sweden). The choice of these suppliers was guided by the availability of these assays in the current hospital routine and availability of resources to acquire additional reagents, and not based on a-priori assumption of higher quality of these assays over other commercially available assays.

From EUROIMMUN, the Systemic Sclerosis [Nucleoli] Profile EUROLINE [IgG]. Antigens tested were: RNA polymerase III (subunits RP11 and RP155), Centromere protein (CenpA and CenpB), Topoisomerase 1 (Scl70), fibrillarin, NOR-90, Th-To, PM-Scl100, PM-Scl75, Ku, tripartite motif-containing protein (TRIM)-21 (Ro 52) and PDGFR. Sera were analyzed at a dilution of 1:100 and autoantibodies were detected using alkaline phosphatase-labeled antihuman IgG. Blots were quantified using the EUROLINE flatbed scanner. Signal intensities of $<11$ intensity units were regarded as negative.

From D-tek, the nitrocellulose blots containing scleroderma associated antigens (SCL10DIV-24) from D-tek (Mons, Belgium) were used. The dot blot contains the antigens Topoisomerase 1 (Scl-70), Centromere protein CenpA and CenpB, PM-Scl75, PM-Scl100, Ku, RNA Polymerase III, Th-To, RNP68/A/C and Fibrillarin. Ten $\mu \mathrm{L}$ of patient serum was used for performing the assay according to manufacturers' protocol. Signal intensities of $<11$ intensity units were regarded as negative.

From Thermo Fischer, the following available (EliA) tests, for Topoisomerase 1 (Scl-70), Ro52, RNA Polymerase III, PM-Scl100, Fibrillarin, Centromere protein (CenpB) were analyzed on a Phadia 250 analyzer according to the manufacturers' protocol. Signal intensities of $<10 \mathrm{U} / \mathrm{ml}$ intensity units were regarded as negative.

\section{Data analysis}

All data were processed and analyzed in the statistical package R (https://www.r-projocts.org), version 3.6.1. Packages for visualization and modelling were tidyr (1.0.0), dplyr (0.8.3) and ggplot2 (3.2.1). Data were used as measured values (autoantibody levels). Autoantibody data were converted to "positive" (present) and "negative" (absent) values using the cut-off values recommended by the manufacturers, i.e. lower limit of normal, recommended by the supplier. The 
concordance between the various assays were calculated and expressed as Cohen's kappa coefficient.

\section{Results}

\section{Prevalence of SSc-specific and -associated autoantibodies}

In total 347 serum samples of individual patients with SSc were evaluated for the presence of SSc autoantibodies (Figure 1(A)). It appeared that there was a difference in prevalence between the autoantibodies in LcSSc and DcSSc patients. The autoantibodies ATA, ARA, fibrillarin and ThTo antibodies revealed a higher prevalence in the diffuse cutaneous form, whereas ACA, Ro52, PM-Scl75, and PMScl100 and $\mathrm{Ku}$ were more prevalent in the limited cutaneous form patients, although for the latter the difference between LcSsc and DcSSc was small. The overall prevalence in the three assays of the antibodies included in the ACR/EULAR classification criteria, namely ACA, ATA, and ARA, ranged between $29.2-30.3 \%, 21.4-24.0 \%$, and $3.8-6.9 \%$, respectively. The prevalence of PM-Scl autoantibodies in the cohort was between 3.2 and $6.4 \%$. Fibrillarin and $\mathrm{Ku}$ autoantibodies could be detected in, respectively, $2.3-3.8 \%$ and $1.4-2.6 \%$ of the patients. Autoantibodies directed against Th-To, NOR90 had a low prevalence $(<2 \%)$ and for PDGFR no positive results were observed. The exact prevalence of autoantibodies per antigen per provider is shown in Table 2.

Subsequently we investigated the prevalence of specific autoantibodies per disease complication group (Figure 1(B)), where we only focussed on the ACA, ATA and ARA autoantibodies. These data showed that ACA autoantibodies associate mainly with PAH and DU complications whereas ATA associates with ILD. The prevalence of ARA is largely comparable in all three complication groups. The data obtained with the assays from all three suppliers showed high agreement.

\section{Concordance between tests}

To assess the agreement between the three assays, we calculated the Cohen's kappa coefficient for the comparisons (Table 3). The concordance for the autoantibodies with the highest prevalence, ATA and ACA, was very high with a Cohen's kappa coefficient of 0.95 , dependent on the specific antigen used, whereas for ARA, a lower concordance of around 0.70 was observed. Using the EUROLINE and EliA assays, respectively 8 and 13 sera were tested positive for the presence of fibrillarin autoantibodies $(k=0.67)$. In

(A)

DcSSc LcSSc

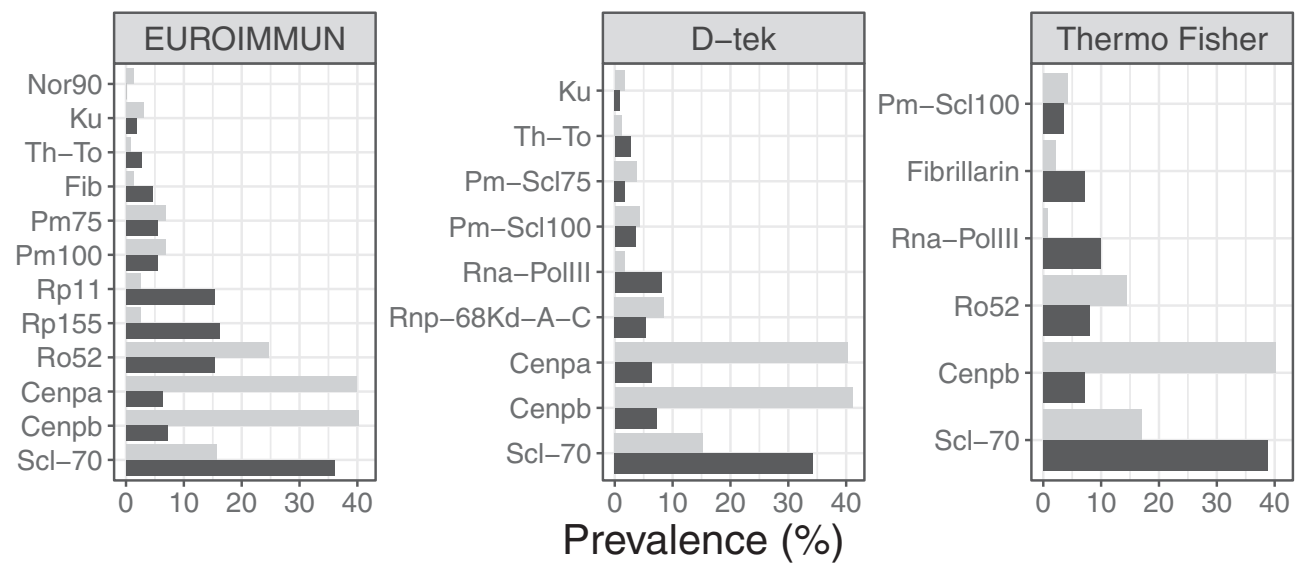

(B)

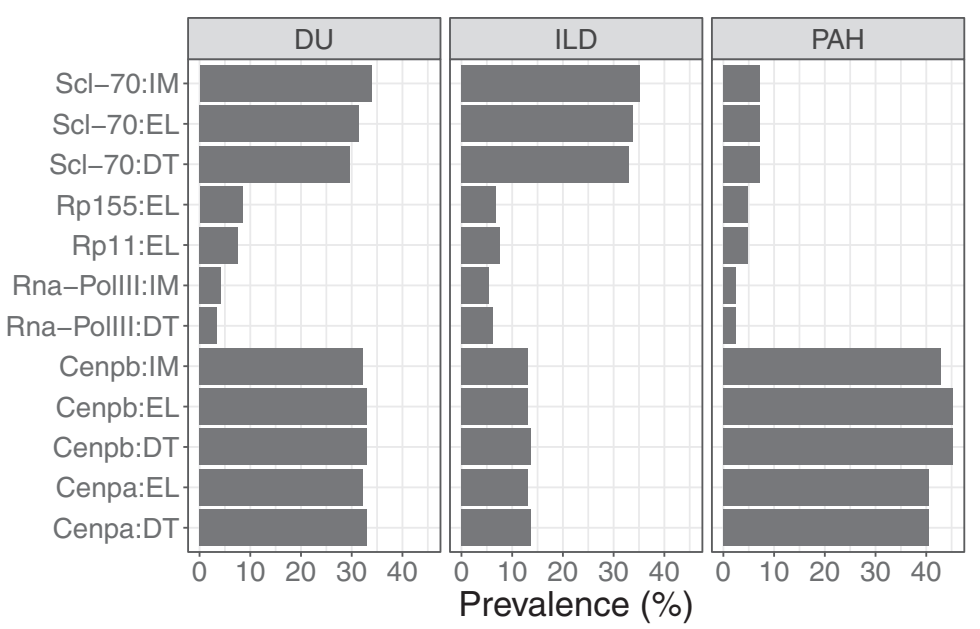

Figure 1. (A) Overview of prevalence of autoantibodies per tested antigen in LCSSC and DcSSC patients. (B) Prevalence of ACA, ARA, and ATA per tested antigen in patient sub-populations with different disease complications. 
Table 2. Overview of prevalence of autoantibodies (per tested antigen) in SSc patients.

\begin{tabular}{|c|c|c|c|c|}
\hline \multirow[b]{2}{*}{ Autoantibody } & \multirow[b]{2}{*}{ Antigen } & \multicolumn{3}{|c|}{ Supplier } \\
\hline & & $\begin{array}{c}\text { EUROLINE } \\
\text { (EUROIMMUN) (\%) }\end{array}$ & $\begin{array}{l}\text { Dot-blot } \\
\text { (D-tek) (\%) }\end{array}$ & $\begin{array}{c}\text { EliA (Thermo Fisher } \\
\text { Scientific) (\%) }\end{array}$ \\
\hline \multirow[t]{2}{*}{ ACA (centromere) } & CENPA & 29.2 & 29.5 & \\
\hline & CENPB & 29.8 & 30.3 & 29.8 \\
\hline ATA (Scl70) & Scl70 & 22.3 & 21.4 & 24.0 \\
\hline \multirow{3}{*}{ ARA (RNA polymerase III) } & Rp11 & 6.6 & & \\
\hline & Rp155 & 6.9 & & \\
\hline & RNAP3 & & 3.8 & 3.8 \\
\hline \multirow[t]{2}{*}{ PM-Scl } & PM75 & 6.4 & 3.2 & \\
\hline & PM100 & 6.4 & 4.0 & 4.0 \\
\hline Fibrillarin & Fibrillarin & 2.3 & ND & 3.8 \\
\hline $\mathrm{Ku}$ & $\mathrm{Ku}$ & 2.6 & 1.4 & \\
\hline Th/To & Th/To & 1.4 & 1.7 & \\
\hline NOR90 & NOR90 & 0.9 & & \\
\hline RNP68/A/C & $\mathrm{RNP} / 68 / \mathrm{A} / \mathrm{C}$ & & 7.5 & \\
\hline PDGFR & PDGFR & 0 & & \\
\hline Ro52 & Ro52 & 21.7 & & 12.4 \\
\hline
\end{tabular}

Prevalence of autoantibodies per provider/test were evaluated using 347 serum samples of individual SSc patients. ND indicates that result was not evaluated due to technical issues with the antigen present in the assay.

Table 3. Concordance between tests.

\begin{tabular}{llc}
\hline Autoantibody & Compared test systems (antigen used in the test) & Concordance (Cohen's $\kappa$ ) \\
\hline ACA & EUROLINE (CENPA) vs. Dot-blot (CENPA) & 0.99 \\
& EUROLINE (CENPB) vs. Dot-blot (CENPB) & 0.99 \\
& EUROLINE (CENPB) vs. EliA (CENPB) & 0.96 \\
& Dot-blot (CENPB) vs. EliA (CENPB) & 0.97 \\
ATA & EUROLINE (Scl70) vs. Dot-blot (Scl70) & 0.94 \\
& EUROLINE (Scl70) vs. EliA (Scl70) & 0.94 \\
ARA & Dot-blot (Scl70) vs. EliA (Scl70) & 0.91 \\
& EUROLINE (Rp11) vs. Dot-blot (RNAP3) & 0.53 \\
& EUROLINE (Rp155) vs. Dot-blot (RNAP3) & 0.57 \\
& EUROLINE (Rp11) vs. EliA (RNAP3) & 0.65 \\
PM-Scl & EUROLINE (Rp155) vs. EliA (RNAP3) & 0.69 \\
& Dot-blot (RNAP3) vs. EliA (RNAP3) & 0.76 \\
& EUROLINE (PM75) vs. Dot-blot (PM75) & 0.59 \\
Fibrillarin & EUROLINE (PM100) vs. Dot-blot (PM100) & 0.77 \\
Ku & EUROLINE (PM100) vs. EliA (PM-Scl100) & 0.77 \\
Th/To & Dot-blot (PM100) vs. EliA (PM-Scl100) & 0.85 \\
Ro52 & EUROLINE (Fibrillarin) vs. EliA (Fibrillarin) & 0.67 \\
\hline
\end{tabular}

The values show the Cohen's kappa coefficient for each comparison.

general, the concordance for the low prevalent SSc-associated autoantibodies, $\mathrm{Ku}$, Th-To and PM-Scl was lower and varied between 0.70 and 0.17 .

\section{Quantitative correlation of read outs}

The analysis described above was based on classification of a sample as either positive or negative, based on the absolute quantitative read out in the antibody assays and the threshold value. We next investigated the correlation between test results based on raw values as an indicator of test characteristics, instead of converting the values to "positive" or "negative" based on the threshold values (Figure 2). The data showed that for the assays for CenpB, which showed a high concordance (Cohen's kappa >0.9), the correlation between the raw values is also high. In contrast, assays that showed low Cohen's kappa coefficient, like $\mathrm{Ku}$ and Th-To and to a lesser extend Fibrillarin, the correlation between the raw values is low or even absent which is related to antigen source or epitope used.

\section{Co-occurrence of autoantibodies in systemic sclerosis}

To see the co-association pattern between the autoantibodies in individual patients, we identified for all autoantibody combinations the level of co-occurrence within individual patients (Figure 3). Based on the percentage overlap, clear clusters (indicated in the blue colour) can be observed for co-associations between the antigens for ACA, ATA, ARA, Ro52, PM-Scl, fibrillarin, in agreement with the high concordance between tests (Table 3 ).

Since ATA, ACA and ARA antibodies are used for disease classification and prognosis, we also examined the possibility of co-occurrence of these SSc-specific autoantibodies with these or other autoantibodies within SSc patients. For this we merged the data from the three suppliers and regarded each patient to be positive for an autoantibody when at least one of the tests showed a positive score. We found that most of the patients (85\%) were positive for only a single antibody, predominantly for ATA $(n=66)$, ACA $(n=63)$ and ARA $(n=23)$. Most double positives (71 in total) were found with Ro52/ACA $(n=21)$, Ro52/RNP- 

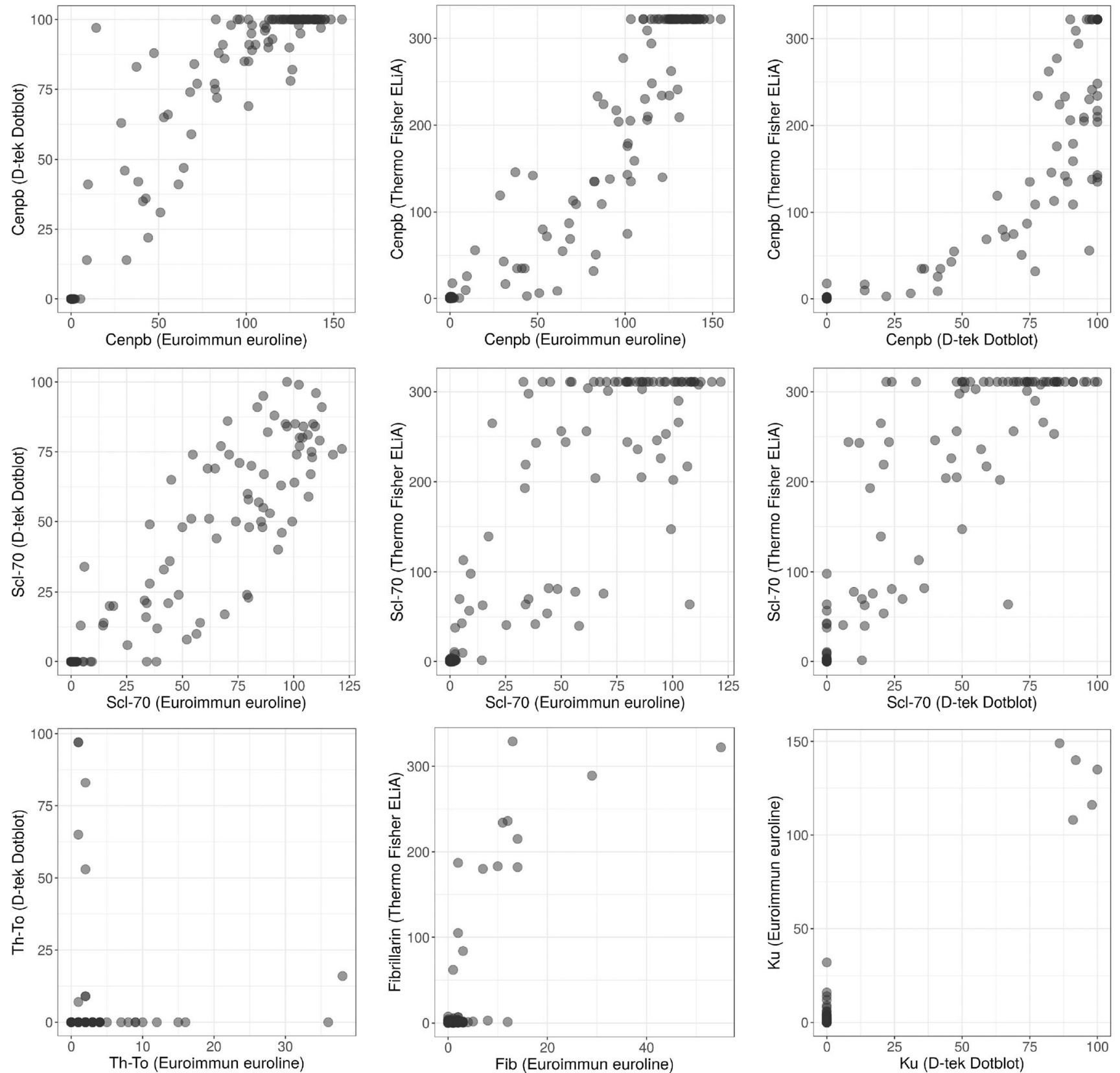

Figure 2. Quantitative comparisons between the tests with equivalent read outs. Examples for the antigens CenpB, Scl-70, Th-To, Fibrillarin, and Ku are shown. The raw values as obtained from the tests from different suppliers are plotted. Results for each assay are expressed in arbitrary units and therefore are not commutable.

$68 \mathrm{Kd}-\mathrm{A}-\mathrm{C}(n=9)$ and Ro52/ATA $(n=8)$. For the major antibodies we found 6 double positives: ATA/ARA (5) and ACA/ATA (1). The combination of ACA/ARA was not found. Multiple triple positives were found with ACA/RNP68Kd-A-C/Ro52 (4). Other triple combinations occurred only once.

\section{Discussion}

Assessment of autoantibody profiles is an integral part of the disease classification in SSc [15]. To date, three autoantibodies, ATA, ARA and ACA, are listed in the ACR/EULAR classification criteria for SSc. These autoantibodies are highly specific for SSc with a specificity of $>95 \%$ for ACA, $>99 \%$ for ATA and $>99 \%$ for ARA, respectively [16]. In the present study, we evaluated the comparability of three different commercially available tests for SSc autoantibodies. Despite technological differences we observed high levels of agreement for both ATA and ACA (Cohen's kappa >0.95). Agreement between assays for ARA and low prevalent SSc autoantibodies was low (a Cohen's Kappa between 0.70 and 0.17 ).

The quality of the diagnostic tests is in this case only inferred from a comparison with each other. It would be interesting to compare these results with an additional nonrelated quantitative measure, such as e.g. ANA immune fluorescence pattern which has been shown to be related to 

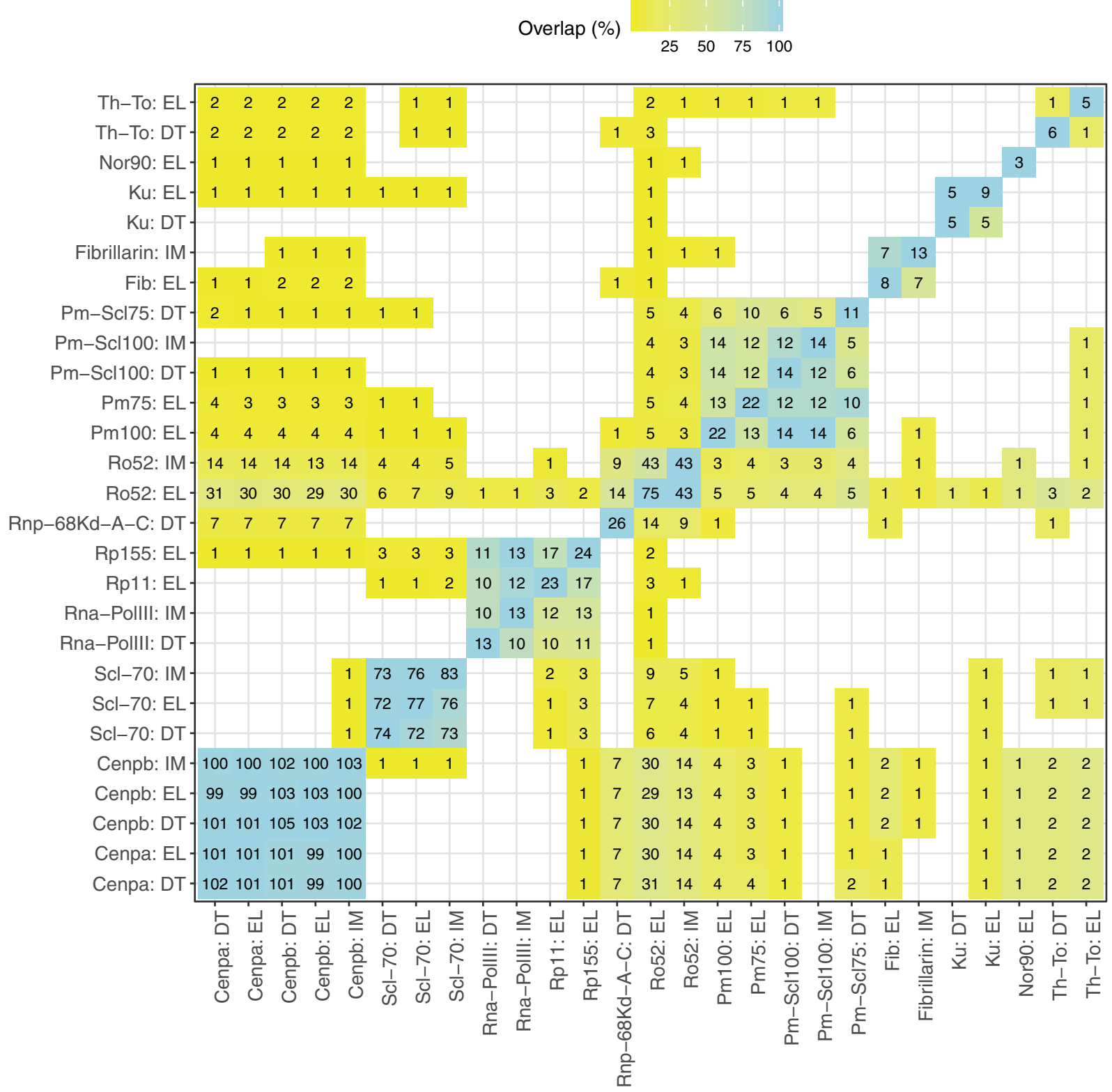

Figure 3. Overlap between all antibodies of three suppliers. The numbers indicate the number of patients and the colour scale indicates the fractional overlap that tested positive for the antibodies indicated on the $x$-axis and $y$-axis. Please note that the calculated percentages are calculated with respect to the set indicated on the $\mathrm{x}$-axis. The percentages may be asymmetrical if the sizes of the sets are unequal. Blank squares indicate a zero overlap.

SSc antibody associations [17]. However, in our cohort we could not make this comparison, since only the qualitative score for ANA-positivity was available.

The diagnostic sensitivity of antibodies in SSc is relatively low because some patients do not have these disease specific autoantibodies, which means that absence of autoantibodies does not rule out SSc. For example, in our cohort $\sim 60 \%$ of the patients was positive for one of these three autoantibodies and $\sim 40 \%$ of the SSc patients had other SSc-specific or - associated autoantibodies (including fibrillarin, PM-Scl, Th-To, NOR-90) or no autoantibodies. Addition of these novel autoantibodies reduced sero-negativity with approximately $12.5 \%$ resulting in an overall sensitivity of both disease-specific and disease-associated antibodies of $72.5 \%$. In other cohorts the percentage of sero-negativity is reported to be around $12 \%$ [18]. This discrepancy in sero-negativity could be due to sample selection, since our cross-sectional cohort is a tertiary referral cohort, including patients in whom the diagnosis of SSc is difficult to make, although they do fulfil the classification criteria.

Our data reveal that there is a high level of agreement between tests from three individual suppliers for ATA and ACA which is reflected in the observed Cohens kappa. The prevalence of ACA in our cohort was approximately $30 \%$, and comparable between the three assays used in our study cohorts and reported elsewhere [18,19]. Of interest, the prevalence of ATA differs between cohorts. We observed a prevalence of $21-24 \%$ depending on the assay used. Other cohort studies report prevalence for ATA of 20-40\% $[18,19]$. This higher prevalence reflects the higher number of more severe cases in our tertiary referral cohort. In our cohort ARA positivity was found in $4-7 \%$ of SSc patients, however, level of agreement was also lower (Cohens Kappa between 0.53 and 0.76 ) which could also contribute to the 
differences in reported prevalence of ARA in cohorts in different countries from 1.5 to $25 \%$ [20]. Also differences of the prevalence dependent on ethnicity have been reported $[21,22]$. Since our cohort is a predominantly Caucasian cohort, a detailed investigation of the relations between prevalence patterns and demographic or ethnic factors was not undertaken.

We found an association of ACA autoantibodies with PAH (45\%, Figure $1(\mathrm{~B}))$ and DU (32\%, Figure $1(\mathrm{~B}))$ and of ATA with ILD (33\% and DU (32\%, Figure 1(B)), which is comparable to published literature $[5,7,23]$. Since our primary aim was the quantitative comparison of three commercial assays, a further analysis of the association patterns with other complications such as GI, joint and muscular involvement was not done.

Though immunoprecipitation is still perceived as the gold standard, more robust and less laborious diagnostic tests have been developed for fast parallel (semi)quantitative detection of autoantibodies. However, antigens used for the detection of autoantibodies differ between the suppliers, preventing a direct quantitative comparison of the results. An additional challenge for harmonization is the absence of antigen specific controls or calibrators. Availability of proficiency testing initiatives, including less prevalent autoantibodies, and sufficient control material together with close collaboration with manufactures could provide a first step in harmonization of these assays [24].

The presence of ARA is associated with rapid diffuse cutaneous disease with poor prognosis and renal crisis and has therefore important clinical consequences [25-28]. Therefore, we can debate whether a concordance between diagnostic ARA assays of $0.76-0.53$ is acceptable. For ACA and ATA the agreement between the tests is sufficient for clinical practice. Results reported on other SSc-associated autoantibodies could be biased, based on the type of assay. Therefore, all types of diagnostic assays should be subject to terms of harmonization and standardization [24]. For autoimmune diagnostics the first step would be to agree on antigen, epitope and alignment of results between different assays. Our results show that this goal is achieved for ACA and ARA autoantibody testing, but for the other SSc-specific and -associated autoantibodies there is room for improvement. There is a clinical unmet need and both a diagnostic and commercial drive to report autoantibodies for diagnostic and prognostic purposes. Furthermore, addressing the issue of the sero-negative gap is warranted. If this harmonization is not achievable, we should rethink how to perform low prevalent autoantibody tests for SSc or how to report the result to our clinicians.

Several studies have examined the co-occurrence of autoantibodies in patients. Combinations of ATA with ACA or ARA have been reported but are rare $[16,18]$. Here we show that the combination of these autoantibodies is dependent on the assay used and is a result of the assay characteristics and antigens used. Because there is no clinical relevance for this co-association, this finding should be interpreted with care as a laboratory finding. Therefore, we should look at the dominant autoantibody in relation to the clinical phenotype.

We conclude that the diagnostic performance of commercially available assays described in this paper for detection of ATA and ACA is good. We found a high concordance between tests. However, concordance of PMScl, RNA Polymerase III and low prevalent autoantibodies like fibrillarin, Th-To and $\mathrm{Ku}$ should be improved. The journey to achieve this important consensus necessitates for laboratory specialists, clinicians and suppliers to join their forces.

\section{Acknowledgements}

We thank EUROIMMUN, D-tek and Thermo Fisher Scientific for providing the reagents for analysis of autoantibodies. Additionally, we thank Esther van Rijssen and Bram van Cranenbroek for their technical assistance. There has been no significant financial support for this work that could have influenced its outcome.

\section{Ethical approval}

The study protocol was reviewed and approved by the local ethics committee. All patients gave written informed consent prior to blood collection.

\section{Disclosure statement}

No potential conflict of interest was reported by the author(s).

\section{ORCID}

Wynand Alkema (D) http://orcid.org/0000-0002-5523-9217

\section{References}

[1] LeRoy EC, Black C, Fleischmajer R, et al. Scleroderma (systemic sclerosis): classification, subsets and pathogenesis. J Rheumatol. 1988;15(2):202-205.

[2] Tyndall AJ, Bannert B, Vonk M, et al. Causes and risk factors for death in systemic sclerosis: a study from the EULAR scleroderma trials and research (EUSTAR) database. Ann Rheum Dis. 2010;69(10):1809-1815.

[3] Kowal-Bielecka O, Landewé R, Avouac J, EUSTAR Co-Authors, et al. EULAR recommendations for the treatment of systemic sclerosis: a report from the EULAR Scleroderma Trials and Research Group (EUSTAR). Ann Rheum Dis. 2009;68(5): 620-628.

[4] Reveille JD, Solomon DH, American G, American College of Rheumatology Ad Hoc Committee of Immunologic Testing Guideline. Evidence-based guidelines for the use of immunologic tests: anticentromere, Scl-70, and nucleolar antibodies. Arthritis Rheum. 2003;49(3):399-412.

[5] Moinzadeh P, Nihtyanova SI, Howell K, et al. Impact of hallmark autoantibody reactivity on early diagnosis in scleroderma. Clin Rev Allergy Immunol. 2012; 43(3) :249-255.

[6] Cabral-Marques O, Riemekasten G. Functional autoantibodies targeting $\mathrm{G}$ protein-coupled receptors in rheumatic diseases. Nat Rev Rheumatol. 2017;13(11):648-656.

[7] Affandi AJ, Radstake TR, Marut W. Update on biomarkers in systemic sclerosis: tools for diagnosis and treatment. Semin Immunopathol. 2015;37(5):475-487.

[8] Graf SW, Hakendorf P, Lester S, et al. South Australian Scleroderma Register: autoantibodies as predictive biomarkers 
of phenotype and outcome. Int J Rheum Dis. 2012;15(1): 102-109.

[9] Domsic RT. Scleroderma: the role of serum autoantibodies in defining specific clinical phenotypes and organ system involvement. Curr Opin Rheumatol. 2014;26(6):646-652.

[10] Mierau R, Moinzadeh P, Riemekasten G, et al. Frequency of disease-associated and other nuclear autoantibodies in patients of the German network for systemic scleroderma: correlation with characteristic clinical features. Arthritis Res Ther. 2011; 13(5):R172.

[11] van den Hoogen F, Khanna D, Fransen J, et al. 2013 classification criteria for systemic sclerosis: an American college of rheumatology/European league against rheumatism collaborative initiative. Ann Rheum Dis. 2013;72(11):1747-1755.

[12] Bonroy C, Van Praet J, Smith V, et al. Optimization and diagnostic performance of a single multiparameter lineblot in the serological workup of systemic sclerosis. J Immunol Methods. 2012;379(1-2):53-60.

[13] van den Hombergh WMT, Carreira PE, Knaapen-Hans HKA, et al. An easy prediction rule for diffuse cutaneous systemic sclerosis using only the timing and type of first symptoms and auto-antibodies: derivation and validation. Rheumatology. 2016; 55(11):2023-2032.

[14] Goh NSL, Desai SR, Veeraraghavan S, et al. Interstitial lung disease in systemic sclerosis: a simple staging system. Am J Respir Crit Care Med. 2008;177(11):1248-1254.

[15] van den Hoogen F, Khanna D, Fransen J, et al. 2013 classification criteria for systemic sclerosis: an American College of Rheumatology/European League against rheumatism collaborative initiative. Arthritis Rheum. 2013;65(11):2737-2747.

[16] Villalta D, Imbastaro T, Di Giovanni S, et al. Diagnostic accuracy and predictive value of extended autoantibody profile in systemic sclerosis. Autoimmun Rev. 2012;12(2):114-120.

[17] Damoiseaux J, Andrade LEC, Carballo OG, et al. Clinical relevance of HEp-2 indirect immunofluorescent patterns: the International Consensus on ANA patterns (ICAP) perspective. Ann Rheum Dis. 2019;78(7):879-889.

[18] Patterson KA, Roberts-Thomson PJ, Lester S, et al. Interpretation of an extended autoantibody profile in a well- characterized Australian systemic sclerosis (scleroderma) cohort using principal components analysis. Arthritis Rheumatol. 2015;67(12):3234-3244.

[19] Liaskos C, Marou E, Simopoulou T, et al. Disease-related autoantibody profile in patients with systemic sclerosis. Autoimmunity. 2017;50(7):414-421.

[20] Meyer OC, et al. Disease subsets, antinuclear antibody profile, and clinical features in 127 French and 247 US adult patients with systemic sclerosis. J Rheumatol. 2007;34(1):104-109.

[21] Mejia Otero C, Assassi S, Hudson M, Genetics versus Environment in Scleroderma Outcome Study, et al. Antifibrillarin antibodies are associated with native North American ethnicity and poorer survival in systemic sclerosis. J Rheumatol. 2017;44(6):799-805.

[22] Rodriguez-Reyna TS, Hinojosa-Azaola A, Martinez-Reyes C, et al. Distinctive autoantibody profile in Mexican Mestizo systemic sclerosis patients. Autoimmunity. 2011;44(7):576-584.

[23] Steen VD. The many faces of scleroderma. Rheum Dis Clin North Am. 2008;34(1):1-15.

[24] Plebani M. Harmonization in laboratory medicine: more than clinical chemistry? Clin Chem Lab Med. 2018;56(10): 1579-1586.

[25] Moinzadeh P, Fonseca C, Hellmich M, et al. Association of anti-RNA polymerase III autoantibodies and cancer in scleroderma. Arthritis Res Ther. 2014;16(1):R53.

[26] Hamaguchi $Y$, Kodera M, Matsushita T, et al. Clinical and immunologic predictors of scleroderma renal crisis in Japanese systemic sclerosis patients with anti-RNA polymerase III autoantibodies. Arthritis Rheumatol. 2015;67(4):1045-1052.

[27] Mouthon L, Bérezné A, Bussone G, et al. Scleroderma renal crisis: a rare but severe complication of systemic sclerosis. Clin Rev Allergy Immunol. 2011;40(2):84-91.

[28] Lazzaroni M-G, Cavazzana I, Colombo E, EUSTAR co-authors, et al. Malignancies in patients with anti-RNA polymerase III antibodies and systemic sclerosis: analysis of the EULAR scleroderma trials and research cohort and possible recommendations for screening. J Rheumatol. 2017;44(5):639-647. 